\section{Intraductal Mucin-Hypersecreting Neoplasm of the Pancreas: Endosonographic Findings}

Mucinous ductal ectasia, also known as intraductal mucin-hypersecreting neoplasm (IMHN), is an uncommon disease in the group of mucinous neoplasms of the pancreas $(1,2)$. It is defined by intraductal growth of neoplastic ductal epithelium, and forms an entity with a spectrum varying from IMHN (without grossly visible tumor) to intraductal papillary neoplasm (without significant mucin production) (3). We report here on the endosonographic findings of papillary proliferation in a case of IMHN of the pancreas.

A 61-year-old man was admitted to hospital due to weight loss, recent diabetic mellitus, and recurrent epigastric pain. His clinical examination was normal, and the laboratory data were normal except for hyperglycemia and steatorrhea. On the CT scan, we noted atrophy of the pancreas, with dilation of the main pancreatic duct (MPD), without calcification. Endoscopic retrograde cholangiopancreatography (ERCP) showed mucus extruding from the major and minor papillae, and filling defects in a dilated MPD without stenosis (Figure 1). Intraductal biopsies showed inflammatory cells, without specificity. On endoscopic ultrasonography (EUS) (Figure 2 ), we noted hyperechoic vegetations in the dilated duct and marked thickening of the duct wall, occurring only in the head of the pancreas. Intraluminal echo-rich material was also seen. The biliary tract and surrounding vessels were normal. Surgical cephalic pancreatectomy was performed, with pancreaticogastric anastomosis. The resected specimen (Figure $\mathbf{3}$ ) showed a cystic dilation in the head of the pancreas, with villous proliferation and mucus. The pathologic diagnosis was papillary epithelial proliferation from the MPD, with severe dysplasia in some areas. Six months after surgery, the patient, under treatment with oral sulfonylurea and pancreatic enzymes, is in good general health.

In contrast to ERCP (4), EUS precisely defined the proliferative intraductal growth as being confined to the head of the pancreas We believe that this type of preoperative information is important in deciding on the type of pancreatectomy to be carried out (Whipple, distal, or total), since this disease can also be multifocal or localized to the tail (5). In addition, EUS has an important role in the preoperative staging, including the assessment of vascular involvement. For the follow-up, a pancreaticogastric anastomosis is probably preferable to a pancreaticojejunal anastomosis, since it makes biopsies, pancreatography, and EUS easier.

\section{A. Glibert', A. Pariente ${ }^{4}$, G. Izard ${ }^{2}$, M. Pellegrin ${ }^{3}$, F. Druart ', T. Morin' \\ ${ }^{1}$ Department of Gastroenterology \\ ${ }^{2}$ Department of Surgery, and \\ ${ }^{3}$ Department of Pathology, Tarbes Hospital, Tarbes, France; \\ ${ }^{4}$ Department of Gastroenterology, Pau Hospital, Pau, France}

\section{References}

1. Warshaw AL, Compton CC, Lewandrowski K, et al:: Cystic tumors of the pancreas: new clinical, radiologic, and pathologic observations in 67 patients. Ann Surg 1990; 212: 432-445.

2. Raijman I, Kortan P, Walden D, et al.: Mucinous ductal ectasia: cholangiopancreatographic and endoscopic findings. Endoscopy 1994; 26: 303-307.

3. Rickaert $F$, Cremer $M$, Devière $J$, et al.: Intraductal mucinhypersecreting neoplasms of the pancreas: a clinicopathological study of eight patients. Gastroenterology 1991; 101: 512-519.
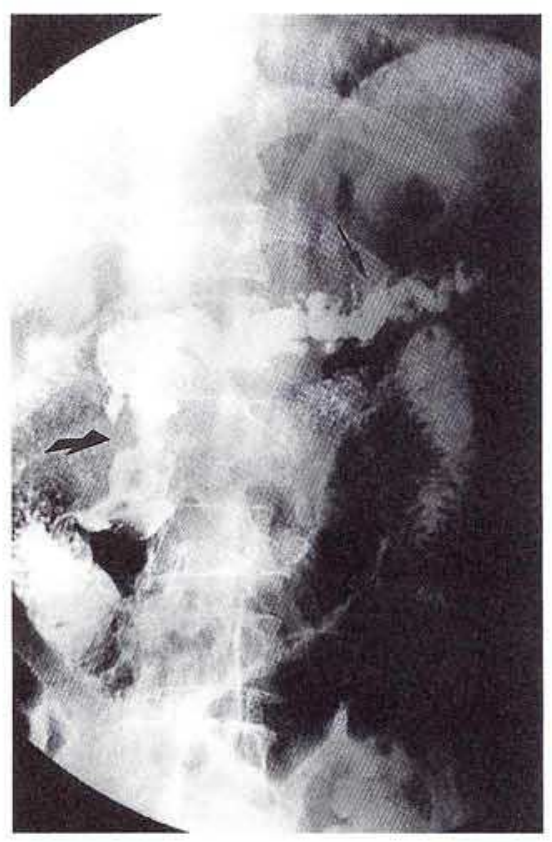

Figure 1: ERCP fluoroscopic view, showing a defect in the marked dilation of the proximal duct in the head of the pancreas (large arrow)

There is an appearance of obstructive pancreatitis above (small arrow).

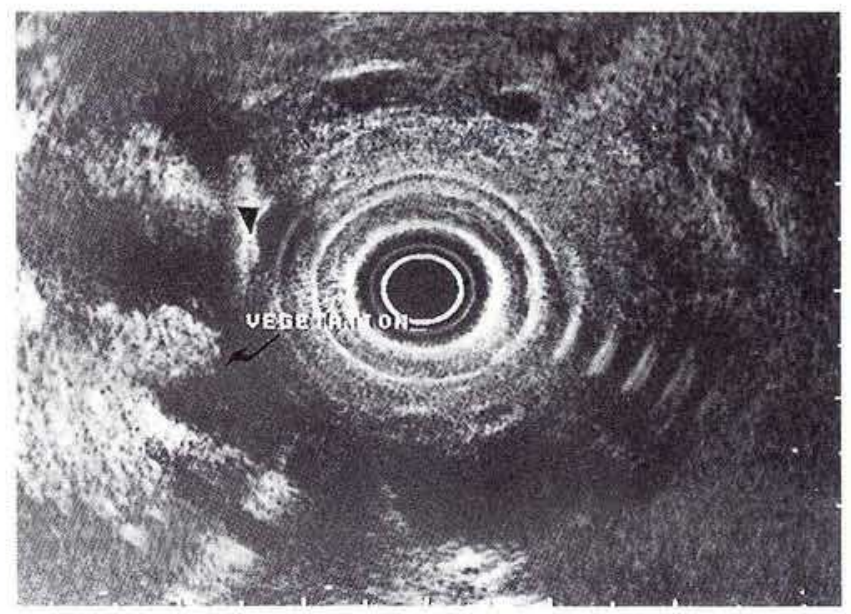

Figure 2: EUS, showing hyperechoic vegetations (arrow) and a hyperechoic defect in the dilated main pancreatic duct (arrowhead)

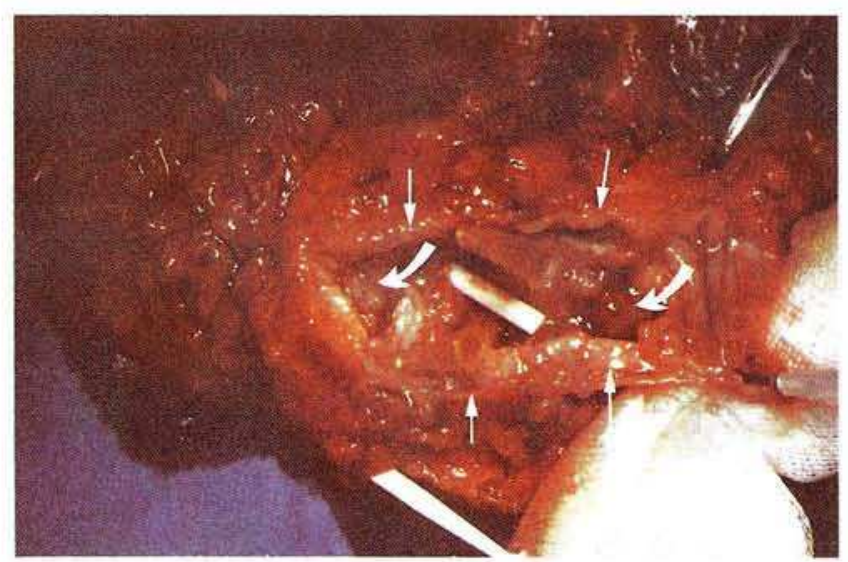

Figure 3: The resected specimen, showing cystic dilation in the head of the pancreas (small arrow), with villous proliferation (large arrow) 
4. Ponsot $P$. Molas $G$, Vilgrain $V$, et al.: Adénomes, adénomatoses et adénocarcinomes pancréatiques intra-canalaires. Gastroenterol Clin Biol 1989; 13: 663-670.

5. Le Berre N. Heresbach D, Robert I, et al.: Les tumeurs mucosécrétantes intra-canalaires du pancréas. Ann Pathol 1994; 14 $87-92$.
Corresponding Author

A. Glibert, M.D.

Service d'Hépato-Gastroentérologic

Centre Hospitalier de Tarbes

Boulevard de Lattre de Tassigny

65013 Tarbes

France

Fax: $+33-62-514893$ 